(C) [2009] IEEE. Reprinted, with permission, from Lu, Zhen., Kadi, Anthony., and Aubrey, Timothy 2009. 'A CME Based Channel Estimation Approach for MIMOOFDM Systems', Proceedings of the 5th International Conference on Wireless communications, networking and mobile computing, pp. 783-786.. This material is posted here with permission of the IEEE. Such permission of the IEEE does not in any way imply IEEE endorsement of any of the University of Technology, Sydney's products or services. Internal or personal use of this material is permitted. However, permission to reprint/republish this material for advertising or promotional purposes or for creating new collective works for resale or redistribution must be obtained from the IEEE by writing to pubs-permissions@ieee.org. By choosing to view this document, you agree to all provisions of the copyright laws protecting it 


\title{
A CME Based Channel Estimation Approach for MIMO-OFDM Systems
}

\author{
Zhen Lu, Anthony Kadi, Tim Aubrey \\ Faculty of Engineering and Information Technology, University of Technology, Sydney \\ Sydney, Australia \\ zlu@eng.uts.edu.au, akadi@eng.uts.edu.au, timaa@eng.uts.edu.au
}

\begin{abstract}
A pilot-assisted, conditional model-order estimation (CME) based channel estimation algorithm is presented. The algorithm is proposed for MIMO-OFDM systems and can detect both channel frequency responses and number of multi-path taps. In addition, the modified CME estimator is also verified its capacity in determining the nonzero taps. The performance of the proposed approach is compared to the popular minimum description length (MDL) algorithm for estimation of the number of channel paths, by means of simulation in the context of a $2 \times 2$ MIMO-OFDM transceiver system. Result indicates that the new algorithm is superior in channel order estimation to the MDL algorithm in MMO-OFDM system over a noisy frequency selective fading channel.
\end{abstract}

Keywords-channel estimation; MIMO; OFDM; space-time coding; CME; MDL; least squares; nonzero taps.

\section{INTRODUCTION}

Orthogonal frequency division multiplexing (OFDM) is a multi-carrier transmission technique. It divides the available spectrum into many closely spaced sub-carriers, resulting in efficient spectrum utilization. The technique also aids in eliminating intersymbol interference (ISI). Multiple-input multiple-output (MIMO) transmission system uses numerous antennas at both the transmitter and receiver, in order to improve system reliability and transmission rate simultaneously. MIMO-OFDM systems have shown great promise in a range of wireless applications and have been adopted in several wireless standards such as IEEE 802.11n, IEEE 802.16e. Such systems are also potential candidates for the fourth-generation (4G) systems [1]

In a MIMO-OFDM system, channel state information (CSI) is essential at the receiver to perform space-time decoding or diversity combining. Various model-based channel estimation approaches have been proposed for single antenna OFDM systems, founded on three main algorithms: maximum likelihood (ML) estimation; least squares (LS) estimation; and minimum mean squared error (MMSE) estimation [2]. In addition, channel estimation approaches can be further categorized as: pilot-assisted [3] - [5] and blind [6] - [8]. Blind techniques increase the effective throughout but either degrade system performance or lower convergence rate. In contrast, the pilot based method consumes more bandwidth, yet simplifies the complexity and improves system performance significantly [9].
In most of the existing estimation schemes for MIMOOFDM there is an assumption that the number of channel taps (i.e. number of propagation paths) is known. In many practical wireless applications in which the propagation environment is both complex and time-variant, this assumption is invalid. Recently, some attention has been directed to estimating the number of multi-path taps. For instance, Yang et. al. described an algorithm for this purpose [10], in which minimum description length (MDL) was first applied in MIMO-OFDM. However, Thomas and Vook found that this algorithm failed when many multi-path arrival times existed in the system, and proposed a near-ML based TOA approach [11]. Wang et. al. documented a probabilistic data association (PDA) based TOA estimation approach for a MIMO-OFDM system [12], which again employed MDL to detect the number of channel paths. Whilst improving performance in channel model estimation, each of these approaches is relatively costly in terms of computational complexity.

In this paper, an efficient modified CME based channel estimation is proposed in MIMO-OFDM system with the purpose of estimating the number of paths in frequency selective fading channels. Given the aim of low computational complexity, least squares (LS) is selected to combine with the modified CME. Moreover, the proposed CME is capable of determining the significant (nonzero) multi-channel taps, and thus has advantage in reducing the complexity as well as improving the efficiency of MIMO system. The rest of this paper is organized as follows: Section II describes a MIMOOFDM system model, the channel model is assumed quasistatic. The proposed scheme is illustrated in section III. In section IV, simulations and numerical results are presented, and the comparison between CME and a MDL based approach is made. Finally, possible improvements and further development are outlined in the conclusion.

\section{A SPACE-TIME CODED MIMO-OFDM MODEL}

In wireless environments, signals bounce off many obstacles such as mountains, buildings, trees, etc. as they propagate between transmitters and receivers. The resultant signal at the receive antenna is, therefore, often the sum of the attenuated transmitted signal and one or more delayed versions of the transmitted signal. This received signal often suffers from intersymbol interference which degrades the signal quality to a certain extent. However, MIMO-OFDM systems 


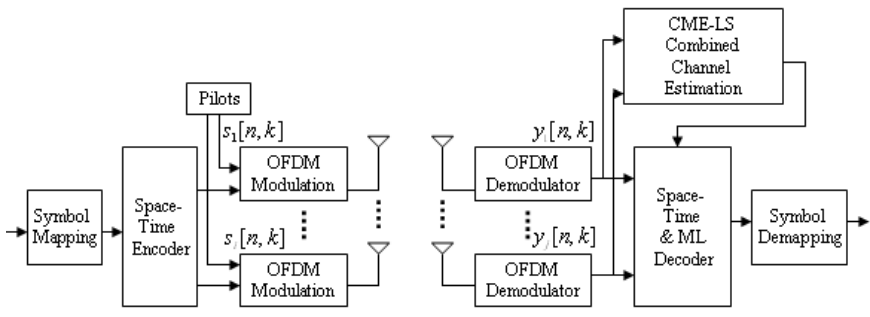

Figure 1. MIMO-OFDM system

are designed to take advantage of the multi-path properties in wireless communications, and are capable of improving transmission rate, range and reliability simultaneously. Fig. 1 depicts a space-time coded MIMO-OFDM system. The system consists of $\mathrm{Nt}$ transmit antennas and $\mathrm{Nr}$ receive antennas. Orthogonal space-time coding (OSTBC) is employed for it can transform the MIMO channel into simple parallel SISO channels. At time $n$, a data block is mapped and encoded into $i$ sequences of information symbols, then pilot symbols are inserted and several different signals $\left(s_{i}[n, k], k=0,1, \ldots K ; i=1\right.$, $2, \ldots N t$ ) can be obtained. $K$ is the number of subchannel, and $i$ represents the ith transmitter antenna. Hence, the output signal of the FFT operation at receiver is expressed as,

$$
y_{j}[n, k]=\sum_{i} H_{i, j}[n, k] s_{i}[n, k]+w_{j}[n, k] .
$$

where $H_{i, j}[n, k]$ is the channel frequency response at the $k$ th tone of the OFDM symbol transmitted from the ith transmit antenna to $j$ th receive antenna at time $n . w_{j}[n, k]$ denotes the additive Gaussian white noise at antenna $j$ with zero mean and variance $\sigma^{2}$. The noise variance $\sigma^{2}$ is assumed unknown in the system. In order to perform decoding and diversity combining at receiver, $H_{i, j}[n, k]$ should be detected. A hybrid CME and LS estimation approach is described in the following section

\section{PROPOSED CME-LS CHANNEL ESTIMATION}

Determination of the number of model parameters is critical in determining an optimal solution in communication systems. An effective approach, channel model order estimator (CME), is proposed in [14]. The algorithm is based on a maximum a posterior (MAP) rule, and can be used to acquire essential parameters in terms of maximizing the conditional probability density function (PDF) of the data, without any requirements of prior knowledge. The CME rule described in [14] chooses the hypothesis that maximizes

$$
L_{X T i}(\mathbf{x})=p_{X}\left(\mathbf{x} ; \boldsymbol{\theta}_{i} \mid H_{i}\right) / p_{T i}\left(\mathbf{T i}(\mathbf{x}) ; \boldsymbol{\theta}_{i} \mid H_{i}\right) .
$$

If the noise variance is unknown, the system can be modeled as a Gaussian linear system,

$$
\mathbf{x}=\mathbf{H} \boldsymbol{\theta}+w,
$$

where $\boldsymbol{H}$ is $N \times p$ matrix, $\boldsymbol{\theta}$ is $p \times 1$ matrix, $\mathrm{w}$ is noise vector $\left\{\mathrm{w} \sim N\left(0, \sigma^{2} \mathbf{I}\right)\right\}$. The complete derivation is illustrated in [14]. Finally, the CME rule is simplified so to choose model order i that minimizes,

$$
\begin{aligned}
& \operatorname{CME}(i)=\ln \widehat{\sigma}_{i}^{2} \times(N-i-2) / 2 \\
& +\frac{1}{2} \ln \left|\mathbf{H}_{i}^{T} \mathbf{H}_{i}\right|+\ln \left\{[\pi(N-i)]^{(N-i) / 2} / \Gamma\left(\frac{N-i}{2}\right)\right]
\end{aligned}
$$

$\mathrm{N}$ is the number of data used to determine the model order, $\mathrm{i}=1$, $2, \ldots, \mathrm{M}, \mathrm{M}$ is the maximum possible order, $\Gamma$ is gamma and $\Gamma(\mathrm{n}+1)=\mathrm{n}$ !, [ ] ${ }^{T}$ stands for transpose of a matrix, and the sufficient statistic for unknown parameters is

$$
\begin{gathered}
\hat{\sigma}_{i}^{2}=\frac{1}{N-i}\left(\mathbf{x}-\mathbf{H}_{\mathbf{i}} \hat{\boldsymbol{\theta}}_{\mathbf{i}}\right)^{T}\left(\mathbf{x}-\mathbf{H}_{\mathrm{i}} \hat{\boldsymbol{\theta}}_{\mathbf{i}}\right), \\
\hat{\boldsymbol{\theta}}=\left(\mathbf{H}^{T} \mathbf{H}\right)^{-1} \mathbf{H}^{T} \mathbf{x} .
\end{gathered}
$$

This paper proposes the use of a modified CME to estimate the number of channel paths in a MIMO-OFDM system.

\section{A. Estimation number of channel paths}

The CME criterion in (2) is documented for Single-input Single-output (SISO) systems. In order to apply the approach in MIMO-OFDM systems and reduce the complexity of the original CME algorithm, pilots are applied in the process of estimation. With the assistance of pilots, $\hat{\boldsymbol{\theta}}$ should be known as ( $\hat{\boldsymbol{\theta}}=\boldsymbol{\theta}$ ), and thus the detection for $\hat{\boldsymbol{\theta}}$ in Equation (6) is unnecessary. According to [14], the sufficient statistic can be defined as

$$
\mathbf{T}\left(\mathbf{y}_{j}\right)=\left\lfloor\mathbf{s}, \hat{\sigma}_{l}^{j^{2}}\right\rfloor .
$$

Using the system mode of section II, the CME criterion is simplified and redefined as,

$$
\begin{aligned}
& p_{X}\left(\mathbf{y}_{j} ; \mathbf{s}, \sigma_{l}^{j^{2}}\right)=\left(2 \pi \sigma_{l}^{j^{2}}\right)^{\frac{-p}{2}} \\
& \times \exp \left[\frac{-1}{2 \sigma_{l}^{j^{2}}}\left(\mathbf{y}_{j}-\sum_{i=1}^{N t} \mathbf{H}_{l}^{i j} s_{i}\right)^{T}\left(\mathbf{y}_{j}-\sum_{i=1}^{N t} \mathbf{H}_{l}^{i j} s_{i}\right)\right] \\
& p_{X}\left(\mathbf{T}\left(\mathbf{y}_{j}\right) ; \mathbf{s}, \sigma_{l}^{j^{2}}\right)=p\left(\mathbf{s} ; \sigma_{l}^{j^{2}}\right) \times p\left(\hat{\sigma}_{l}^{j^{2}} ; \sigma_{l}^{j^{2}}\right) \\
& =\left[(2 \pi)^{P}\left|\sigma_{l}^{j^{2}}\left(\mathbf{H}_{l}^{i j} \mathbf{H}_{l}^{i j}\right)^{-1}\right|\right]^{-1 / 2} \times\left[(P-l) \hat{\sigma}_{l}^{j^{2}} / \sigma_{l}^{j^{2}}\right]^{(P-l) / 2-1} \\
& \times \exp \left[\frac{-(P-l)}{2 \sigma_{l}^{j^{2}}} \hat{\sigma}_{l}^{j^{2}}\right] \cdot\left\{(P-l) /\left[\sigma_{l}^{j^{2}} \times 2^{(P-l) / 2} \Gamma\left(\frac{P-l}{2}\right)\right]\right\} \\
& \left(\widehat{\sigma}_{l}^{j}\right)^{2}=\frac{1}{P-l}\left(\mathbf{y}_{j}-\sum_{i=1}^{N t} \mathbf{H}_{l}^{i j} S_{i}\right)^{T}\left(\mathbf{y}_{j}-\sum_{i=1}^{N t} \mathbf{H}_{l}^{i j} S_{i}\right) .
\end{aligned}
$$

Therefore, from (2), (7), (8), (9), we can get

$$
\begin{aligned}
& L_{X T i}(y)=p_{X}\left(\mathbf{y} ; \mathbf{s}, \sigma_{l}^{j}\right) / p_{T i}\left(\mathbf{T i}(\mathbf{y}) ; \mathbf{s}, \sigma_{l}^{j}\right) \\
& =\Gamma\left(\frac{P-l}{2}\right) /\left\{[\pi(P-l)]^{(P-l) / 2}\left|\mathbf{H}_{l}^{i j T} \mathbf{H}_{l}^{i j}\right|^{1 / 2}\left(\hat{\sigma}_{l}^{j^{2}}\right)^{(P-l) / 2-1}\right\}
\end{aligned}
$$

Equation (11) can be finally expressed as

$$
\begin{aligned}
& \operatorname{CME}_{\mathrm{ij}}(l)=[(P-l-2) / 2] \times \ln \left(\hat{\sigma}_{l}^{j}\right)^{2} \\
& +\frac{1}{2} \ln \left|\left(\mathbf{H}_{l}^{i j}\right)^{T} \mathbf{H}_{l}^{i j}\right|+\ln \left\{[\pi(P-l)]^{(P-l) / 2} / \Gamma\left(\frac{P-l}{2}\right)\right\}
\end{aligned}
$$

where $\left(\hat{\sigma}_{l}^{j}\right)^{2}$ is given in (9), $\mathbf{H}_{l}^{i j}$ is the estimated channel frequency response between ith transmitter antenna and $j$ th receiver antenna obtained by LS estimation, $s_{i}$ are the pilots delivered from ith antenna, $P$ is the total number of pilots and $l$ $=1,2, \ldots, \mathrm{L}, \mathrm{L}$ stands for maximum possible number of 
channel paths. Therefore, the number of channel paths between ith transmitter and $j$ th receiver antenna can be finally detected by minimizing CME,

$$
\hat{l}=\arg \min _{l}\left\{C M E_{i j}\left(\mathbf{y}_{j}, l\right)\right\} \text {. }
$$

In order to acquire the number of channel taps, the channel frequency response is required. Here a least squares (LS) approach is used for the purpose due to its low computational complexity. However the proposed CME can be combined with other channel detection methods such as minimum mean square error (MMSE), which are capable of achieving better performance.

\section{B. Pilot assisted LS estiamtior}

The frequency selective channel is assumed to be Rayleighfading, with frequency response: $H_{i, j}[n, k]$ and impulse response: $h_{i, j}[n, l], l=0,1, \ldots, L-1$, corresponding to the channel between ith transmit-antenna and $j$ th receive-antenna, and $L$ is the number of channel taps. The received pilot symbols at the $j$ th receive antenna are,

$$
y_{j}[n, p]=\sqrt{\varepsilon_{p}} \sum_{i} \mathbf{H}_{i j}[n, p] s_{i}[n, p]+w_{j}[n, p], p \in I_{p},
$$

where $w_{j}[n, p]$ is additive white Gaussian noise (AWGN), $\varepsilon_{p}$ is the transmitted power per pilot symbol, $I_{p}$ denotes the position of pilot sub-carriers, and $P$ is the number of pilots. Using [15] the following is defined

$$
\begin{aligned}
\mathbf{G}_{i}= & \left(\varepsilon_{p} \mathbf{F}_{p} \mathbf{D}^{H}\left(s_{i}[n, p]\right) \mathbf{D}\left(s_{i}[n, p]\right) \mathbf{F}_{p}^{H}\right)^{-1} \\
& \times\left(\sqrt{\varepsilon_{p}} \mathbf{D}\left(s_{i}[n, p]\right) \mathbf{F}_{p}^{H}\right)^{H}
\end{aligned}
$$

and the LS estimate of channel impulse is given by

$$
\hat{h}_{i j}=\mathbf{G}_{i} y_{j}[n, p]
$$

where $\mathbf{F}$ is a $L \times K$ matrix $[\mathbf{F}]_{l, k}=\exp (j 2 \pi(l-1)(k-1) / K)$, $\mathbf{f}_{i p},\left(\{i p\}_{i p=1}^{p}=I_{p}\right)$ is $p$ th column of $\mathbf{F}, \mathrm{F}=\left[\boldsymbol{f}_{1}, \ldots, \boldsymbol{f}_{i p}, \ldots, \boldsymbol{f}_{p}\right]$, and $s_{i}[n, p]$ is the transmitted pilot symbols. In addition, $\boldsymbol{D}(x)$ stands for a diagonal matrix with $x$ in its diagonal, and []$^{H}$ is the Hermitian transpose. Thus, the estimated channel frequency response on the $n$th subcarrier is

$$
\hat{H}_{i j}(n)=f_{p}^{H} \hat{h}_{i j} .
$$

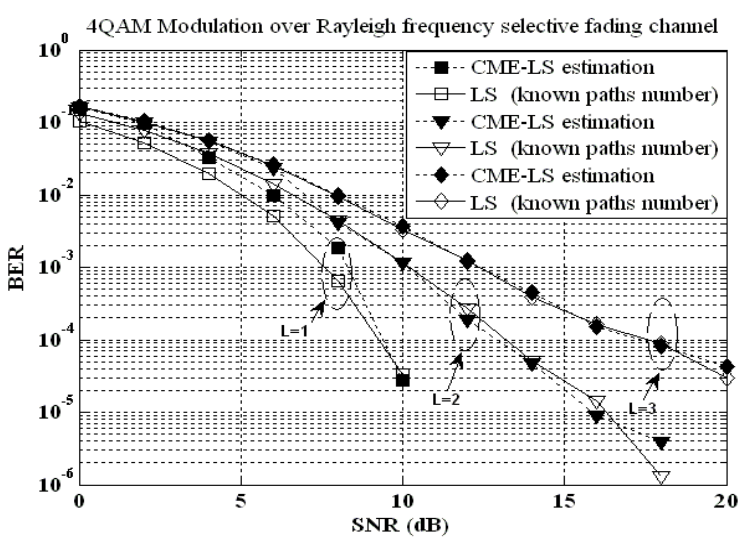

Figure 2. Comparison of BER performance

\section{SIMULATION RESULTS}

A $2 \times 2$ MIMO-OFDM system with Alamouti space-time code [13], which is the best known OSTBC, is employed to demonstrate the performance of proposed hybrid approach. In simulation, parameters of the system are set according to IEEE 802.16 (2004), each OFDM symbol contains K sub-carriers $(\mathrm{K}=256)$ in which 192 tones are the used for data transmission and $\mathrm{P}(\mathrm{P}=8)$ pilots are utilized to detect both channel taps and channel gains, the remaining sub-carriers are employed as lower and upper guards. The cyclic prefix (CP) number is chosen as $\mathrm{G}=\mathrm{K} / 4=64$. The system bandwidth is $1.75 \mathrm{MHz}$, and the sampling factor is $8 / 7$. Since 256 sub channels are used, the useful symbol time is $128 \mu \mathrm{s}$ and $\mathrm{CP}$ time is $32 \mu \mathrm{s}$. With the aim of testing the proposed approach, the assumption that the maximum delay of multi-path fading channels is less than the cyclic prefix period (32 $\mu$ s), and can thus be ignored, is made. A comparison of BER performance for 4QAM modulation, between LS estimation with known number of taps and CMELS estimation without any CSI is depicted in Fig.2.

The figure shows that there is only around $1 \mathrm{~dB}$ performance degradation for CME-LS estimation while the number of channel paths $\mathrm{L}=1$. However, for channels with 2 and 3 paths, the BER performances of the CME-LS algorithm are almost the same as the detection with known number of channel paths. It should be noted that there is an upper limit to the number of taps that can be accurately estimated based on the number of pilot tones used. According to [10], in order to correctly detect $\mathrm{L}$, the number of pilots $P$ should be: $P \geq 2 \mathrm{~L}+1$, and thus no more than 3 channel paths can be detected in the simulation above since only 8 pilot symbols are employed. Using the same simulation parameters as above and in the case of $\mathrm{SNR}=20 \mathrm{~dB}$, an accuracy of only around $30 \%$ is achieved for $L=4$ (See Fig. 3). Increasing the number of pilots will increase the estimation accuracy for more complex propagation environments, but will also reduce bandwidth efficiency. In many common propagation environments, it may be possible to assume that there are only 3 or less dominant transmission paths. Where such an assumption does not hold, more pilots should be employed, despite the consumption of additional bandwidth.

The performance of CME and minimum description length (MDL) is also compared through simulation. MDL has been employed in MIMO-OFDM system in [10] and [12]. According to [14], the MDL for MIMO system can be expressed as,

$$
\begin{gathered}
M D L_{i j}(l)=(P / 2) \ln \left(\hat{\sigma}_{l}^{j}\right)^{2}+[(L+1) / 2] \ln P \\
\left(\hat{\sigma}_{l}^{j}\right)^{2}=\left(y_{j}-\sum_{i} \mathbf{H}_{l}^{i j} S_{i}\right)^{T}\left(y_{j}-\sum_{i} \mathbf{H}_{l}^{i j} s_{i}\right) / N \\
\hat{l}=\arg \min _{l}\left\{M D L_{i j}(\mathbf{y}, l)\right\}
\end{gathered}
$$

The comparison between CME and MDL is given in terms of accuracy rate, which defines as,

$$
\text { accuracy rate }=\frac{\text { number of correct detection }}{\text { total number of detection }} \times 100 \%
$$

Fig. 3 indicates the comparison of accuracy rate between CME and MDL at different SNR. The results show that the 
CME is superior to MDL in estimation of the number of channel paths. According to the analysis above, while the $\mathrm{L} \geq 4$, the 8 pilot estimator is insufficient. For a more complex wireless environment 16 pilot symbols are used and accuracy results are given in Table I. In this case, more pilots are utilized and the number of channel paths that can be correctly detected increases. A comparison of both estimators is shown in Fig.3. It is noted that CME outperforms MDL.

In practice, wireless multi-path channels are dominated by a relatively small number of significant paths, and detecting nonzero taps becomes a critical approach in reducing algorithm complexity and improving system efficiency. The proposed approach is also verified as an effective method for determining the number of key taps in a MIMO-OFDM system. Examples obtained from the simulation with 8 pilots are shown in Table II. A 100 packet of data is utilized as input signal, the actual number of multi-path taps is set to 3 , with one of the paths having a much lower power than the others. $\left(P_{h i}=\left|h_{i}\right|^{2}<2|w|^{2},|w|^{2}\right.$ is noise power, $\left.i=1,2,3\right)$. According to Table II, the majority of the packets only detect two paths in spite of three taps being present in the system (e.g. SNR=20dB, 99 out of 100 packets determine that the number of taps is 2). The performance of the proposed CME improves significantly as noise decreases.

\section{CONCLUSION}

In this paper, the proposed pilot-assisted CME based channel estimation algorithm has been shown to be a viable approach in determining the number of channel paths, especially nonzero paths, for MIMO-OFDM systems in the absence of prior knowledge of model parameters. A comparison of the performance between the modified CME and MDL has shown that the former is superior. Although the simulations have been done in relatively simple wireless environments, i.e. the number of channel taps is less than or equal to 3, it is possible to apply the estimation scheme in more complex environments by increasing the number of pilot tones. While the modified CME algorithm is combined with LS channel estimation, it is not restricted to this approach and can be combined with other estimation schemes. For example, a hybrid MMSE-CME algorithm could improve the system BER performance at the cost of increased computational complexity. The benefit of the CME approach to estimating the number of channel paths has been established. Further research into hybrid methods employing this approach is warranted.

\section{TABLE I. COMPARISON OF CME \& MDL WITH 16 PILOTS}

\begin{tabular}{|c|c|c|c|c|c|c|c|}
\hline & & \multicolumn{5}{|c|}{ Actual Model Order (L) } \\
\hline SNR=20dB & Algorithm & 1 & 2 & 3 & 4 & 5 & 6 \\
\hline \multirow{2}{*}{ Accuracy Rate } & CME & $75.70 \%$ & $82.45 \%$ & $82.70 \%$ & $88.85 \%$ & $76.60 \%$ & $71.40 \%$ \\
\cline { 2 - 7 } & MDL & $58.50 \%$ & $74.65 \%$ & $77.90 \%$ & $87.60 \%$ & $71.95 \%$ & $64.55 \%$ \\
\hline
\end{tabular}

TABLE II. CME IN NONZERO TAPS DETECTION

\begin{tabular}{|c|c|c|c|c|c|}
\hline & \multirow{2}{*}{ SNR(dB) } & \multicolumn{4}{|c|}{ Model Order under Estimation (I) } \\
\cline { 3 - 6 } & & 1 & 2 & 3 & 4 \\
\hline Number of Estimated & 10 & 5 & 72 & 20 & 3 \\
\cline { 2 - 6 } Order $\left(N_{\hat{l}=l}\right)$ & 15 & 2 & 90 & 8 & 0 \\
\cline { 2 - 6 } & 20 & 0 & 99 & 1 & 0 \\
\hline
\end{tabular}
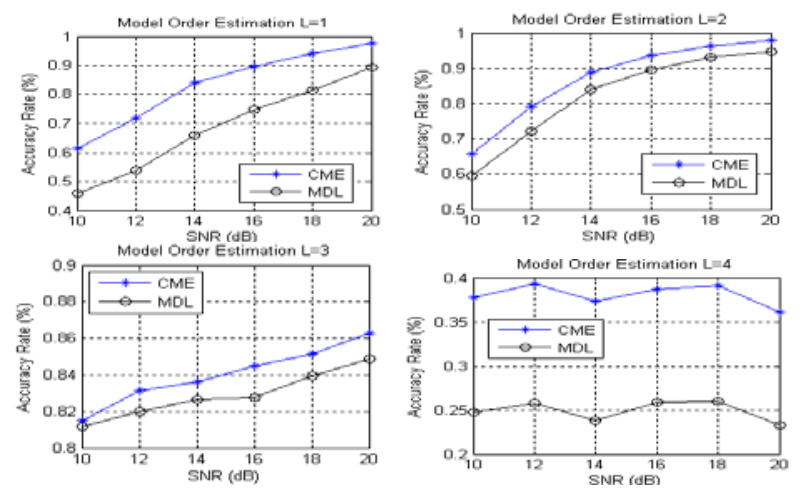

Figure 3. Comparison of accuracy rate with 8 pilots

\section{REFERENCES}

[1] G. L. Stuber, J. R. Barry, S. W. McLaughlin, Y. Li, M. A. Ingram and T. G. Pratt, "Broadband MIMO-OFDM wireless communications", Proceedings of the IEEE, vol. 92, no. 2, pp. 271-294, 2004

[2] S. M. Kay, Fundamentals of Statistical Signal Processing: Estimation Theory, PTR Prentice-Hall, Englewood Cliffs, N.J, 1993.

[3] J. K. Cavers, "An analysis of pilot symbol assisted modulation for Rayleigh fading channels [mobile radio]”, Vehicular Technology, IEEE Transactions on, vol. 40, no. 4, pp. 686-693, 1991

[4] Y. Li, N. Seshadri, and S. Ariyavisitakul, "Channel estimation for OFDM systems with transmitter diversity in mobile wireless channels”, Selected Areas in Communications, IEEE Journal, vol. 17, no. 3, pp. 461-471, 1999

[5] Y. Li, "Simplified channel estimation for OFDM Systems with multiple transmit antennas”, Wireless Communications, IEEE Transactions on, vol. 1, No. 1, pp67-75, 2002

[6] Z. Ding, and Y. Li. Blind channel equalization and identification, Marcel Dekker, New York, 2000.

[7] M. C. Necker and G. L. Stüber, "Totally blind channel estiamtion for OFDM on fast varying monile radio channels," IEEE Trans. Wireless Commun., vol 3, no. 5, pp. 1514-1525, Sep. 2004.

[8] I. Bradaric, A. P. Pertropulu and K. I. Diamantaras, "Blind MIMO FIR channel identification based second-order spectra correlations," IEEE Trans, Signal Process., vol. 51, no. 6, pp. 1668-1674, Jun. 2003.

[9] M. Jiang and L.Hanzo, "Multiuser MIMO-OFDM for next-generation wireless systems,”Processings of the IEEE, vol. 95, no. 7, July, 2007

[10] B. Yang, K.B. Letaief, R.S. Cheng and C. Zhigang. "Channel estimation for OFDM transmission in multipath fading channels based on parametric channel modelling”, Communications, IEEE Transactions on, vol. 49, no. 3, pp. 467-479, 2001

[11] T. A. Thomas and F. W. Vook. "Broadband MIMO-OFDM channel estimation via near maximum likelihood time of arrival estimation", Acoustics, Speech, and Signal Processing, 2002. Proceedings. (ICASSP '02). IEEE International Conference on, vol. 3, pp. III-2569-III-2572 vol.2563, 2002

[12] Z. J. Wang, H. Zhu and K. J. R. Liu. "A MIMO-OFDM channel estimation approach using time of arrivals”, Wireless Communications, IEEE Transactions on, vol. 4, no. 3, pp. 1207-1213, 2005

[13] S. M. Alamouti. “A simple transmit diversity technique for wireless communications", Selected Areas in Communications, IEEE Journal on, vol. 16, no. 8, pp. 1451-1458, 1998.

[14] S. Kay. "Conditional model order estimation”, Signal Processing, IEEE Transactions on, vol. 49, no. 9, pp. 1910-1917, 2001.

[15] X. Cai \& G .B. Giannakis. "Error probability minimizing pilots for OFDM with M-PSK modulation over Rayleigh-fading channels”, Vehicular Technology, IEEE Transactions on, vol. 53, no. 1, pp. 146155, 2004 